\title{
Critical phenomena of chain-molecule solutions
}

\author{
Xueqin An, Fuguo Jiang, Chuanyin Chen, Weiguo Shen ${ }^{\bullet}$ \\ Department of Chemistry, Lanzhou University, Lanzhou, Gansu 730000, P.R. China
}

\begin{abstract}
A model of Landau-Ginsburg-Wilson type is used to describe the dependence of the critical amplitude (associated with coexistence curve, or correlation length, or susceptibility) on molar mass for chain-molecule solutions of both small molecules and polymers. Experimental methods for measurements of the critical volume fraction, coexistence curve, and turbidity are discussed. Some experimental results are summarized and compared with the theory, which shows that the theory is satisfied within experimental uncertainties.
\end{abstract}

\section{INTRODUCTION}

It is well known that polymer solutions near their critical consolute points exhibit behaviour similar to those of binary mixtures of small molecules, pure fluids, and ferromagnets in the critical regions. They fall into the same universality class of the 3-D Ising model. Like the other numbers of this class, the various thermodynamic properties of critical polymer solutions show the power-law dependence on the distance in temperature from the critical point. In addition, the amplitudes of the power-law expressions are dependent on the number of the units in the polymer, or the molar mass of the polymer (ref.1- ref.5). These can be described as the general forms:

$$
\begin{aligned}
& \Delta \phi=B|t|^{\beta} \propto M^{-b}|t|^{\beta} \\
& \xi=\xi_{0}|t|^{-\nu^{\prime}} \propto M^{n}|t|^{-\nu} \\
& \chi=\chi_{0}|t|^{-\gamma} \propto M^{g}|t|^{-\gamma}
\end{aligned}
$$

with $t=\left(T-T_{c}\right) / T_{c}$, where $\Delta \phi, \xi, \chi$, and $T_{c}$ are difference of volume fractions of two coexisting phases, correlation length, susceptibility, and the critical temperature, respectively; $\beta, \nu$, and $\gamma$ are $t$ exponents, and well established as universal indices by experiments and theories. The coefficients $B, \xi_{0}$, and $\chi_{0}$ in equation (1) are defined as the amplitudes that are dependent on $M$. This dependence may be characterized by $M$ exponents $b, n$, and $g$, and the values of $M$ exponents are generally believed to be universal.

Some theories such as de Gennes approximation (ref.6), Landau-Ginsburg-Wilson (LGW) model (ref.7), and scaling laws (ref.5) have been developed to explain the experimental results. Experimentally, precise data available for testing the universality are quite limited. TABLE 1 lists some values of $M$ exponents from theories and experiments. Somewhat molar mass dependence of $M$ exponents has been detected (ref.4, ref. 8 ), which results in large uncertainties in $M$ exponents. Therefore, the disagreements between different

\footnotetext{
- to whom the corespondence should be addresed
} 
theories and experiments are difficult to be distinguished by the available experimental results. Both of the theoretical and experimental studies are expected to explore the $M$-dependence critical phenomena in polymer solutions more precisely. In addition, most of the theories to describe the $M$ dependence of the critical amplitudes started with Flory-Huggins theory, which has been also widely used in predicting the properties of the mixtures of small molecules containing $n$-alkanes with various chain lengths. Therefore, in principle, the theories to describe $M$ dependence of the critical amplitudes for polymer solutions should also be valid to the mixtures of small chain molecules, provided the limitation of $\phi_{c} \rightarrow 0$ is abandoned.

TABLE 1. Comparison of Experimental and Theoretical Values of the MExponents.

\begin{tabular}{cccccc}
\hline M-exponent & $\begin{array}{c}\text { experimental } \\
\text { value }\end{array}$ & $\begin{array}{c}\text { classical } \\
\text { value }\end{array}$ & $\begin{array}{c}\text { de Gennes } \\
\text { approx. }\end{array}$ & LGW model & scaling laws \\
\hline$b$ & $0.23 \sim 0.34^{\mathrm{a}}$ & $1 / 4$ & 0.337 & 0.27 & 0.285 \\
$n$ & $0.13 \sim 0.28^{b}$ & $1 / 4$ & 0.185 & 0.17 & 0.195 \\
$g$ & $0.03 \sim 0.28^{\mathrm{c}}$ & 0 & -0.12 & -0.03 & 0.025 \\
\hline
\end{tabular}

${ }^{\mathrm{a}} \mathrm{See}\left(\right.$ ref.4). ${ }^{\mathrm{b}} \mathrm{See}$ (ref.8). ${ }^{\mathrm{c}} \mathrm{See}$ (ref.5).

Recently, we derived the universal dependence of the critical amplitudes (associated with coexistence curve, correlation length, and susceptibility) on molar mass from a model of Landau-Ginsburg-Wilson type for chain-molecule solutions of both small molecules and polymers (ref.3). These relations may be written as:

$$
\begin{aligned}
& \left.\Delta \phi\left(1-\phi_{c}\right)^{-1.865} \propto M^{-0.077}\left\{\phi_{c} /\left(1-\phi_{c}\right)\right\}^{0.519} \mid t\right\}^{0.327} \\
& \xi\left(1-\phi_{c}\right)^{0.85} \propto M^{0.13}\left\{\phi_{c} /\left(1-\phi_{c}\right)\right\}^{-0.11}|t|^{-0.63} \\
& \chi\left(1-\phi_{c}\right)^{-1.20} \propto M^{0.24}\left\{\phi_{c} /\left(1-\phi_{c}\right)\right\}^{0.72}|t|^{-1.24}
\end{aligned}
$$

where, $\phi_{c}$ is the critical mole fraction. The universality of equation (2) may be tested by the experimental studies on the critical behaviours of various chain-molecule solutions of both small molecules and polymers.

\section{EXPERIMENTAL METHODS}

\section{Determination of the critical yolume fraction}

The critical volume fractions may be determined by the technique of "equal volumes of two coexisting phases" (ref.9, ref.10). A sample was prepared in a glass tube of $10 \mathrm{~mm}$ id. provided with an Ace-thread

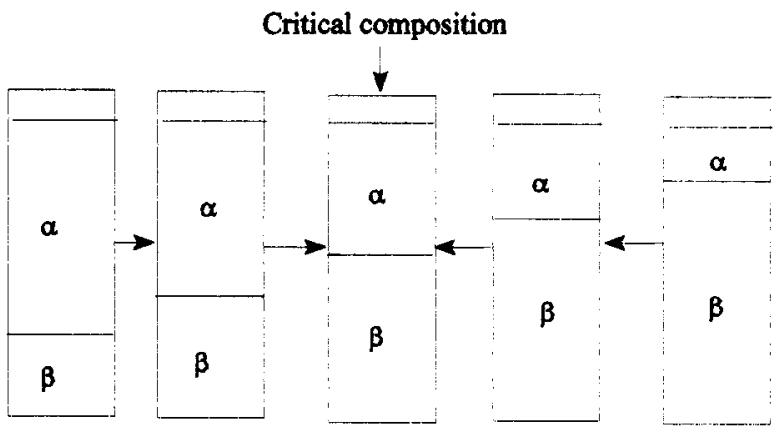

Fig. 1 The procedure to determine the critical composition connector, which allowed it to be sealed with a Teflon cap. The mole fraction was determined by weighing the two components added into the sample cell. The sample tube was placed into a water bath, in which temperature could be controlled to within $\pm 0.002 \mathrm{~K}$. After phase equilibrium had been reached, the height of each phase was measured at several temperatures with a cathetometer and the height fraction (approximately equals to the volume fraction of two phases) was calculated. The critical composition was estimated from the height fraction, and a new sample with the estimated composition was prepared and studied. This procedure was repeated until approximately equal volumes of two phases were obtained. The procedure to search the critical composition is shown schematically in Fig. 1. The precision in determination of the critical mole fraction is about 0.001 and the critical volume fraction may be converted from the critical mole fraction with known densities of two components. 


\section{Determination of the coexistence curve}

A sample with critical composition was prepared in a rectangular fluorimeter cell. The refractive indices of two coexisting phases in the cell were measured at various temperatures by the method of "minimum deviation" (ref. 10, ref.11). A schematic diagram of the apparatus is shown in Fig. 2. The angle of minimum $\Phi$ was calculated by iteratively solving the following

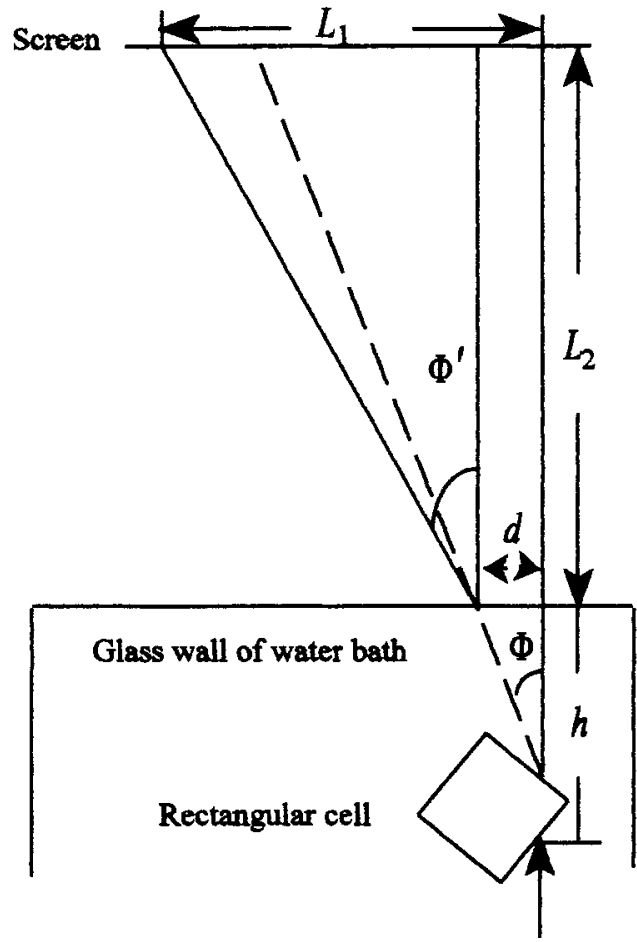

Light beem

Fig. 2 Schematic diagram of apparatus for measurement of the coexistence curve

$$
\begin{aligned}
& \left(L_{1}-d\right) / L_{2}=\tan \Phi \\
& d=h \tan \Phi \\
& \sin \Phi^{\prime}=n_{\mathrm{w}} \sin \Phi
\end{aligned}
$$

where $n_{w}$ is the refractive index of water related to air, and $d, h, L_{1}, L_{2}, \Phi$, and $\Phi^{\prime}$ are defined by Fig. 2 . The refractive index of the sample is obtained by

$$
n_{s}=n_{w}\{\sin [(\pi / 2+\Phi) / 2]\} / \sin (\pi / 4)
$$

The precision of measurement of the refractive index was about \pm 0.0001 . In order to convert the coexistence curve $(T, n)$ to curves $(T, x)$ and $(T, \phi)$, a series of samples of binary mixtures with known compositions was prepared and their refractive indices in the onephase region at various temperatures were measured, which gave the dependence of refractive index on the temperature and the mole fraction (or the volume fraction)(ref.10).

\section{Turbidity measurement}

Correlation length and susceptibility are related to turbidity by the following equation (ref.9):

$$
\tau=\left(\pi^{3} / \lambda_{0}^{4}\right)\left(\partial n^{2} / \partial \phi\right)^{2} k_{b} T \chi \mathrm{f}(\alpha)
$$

where, $\lambda_{0}$ is the wavelength of light in a vacuum, $k_{b}$ is Boltzmann's constant, $n$ is the refractive index of the solution. The correlation length enters through the function $f(\alpha)$ :

$$
f(\alpha)=\left(2 \alpha^{2}+2 \alpha+1\right)[\ln (1+2 \alpha)] / \alpha^{3}-2(1+\alpha) / \alpha^{2}
$$

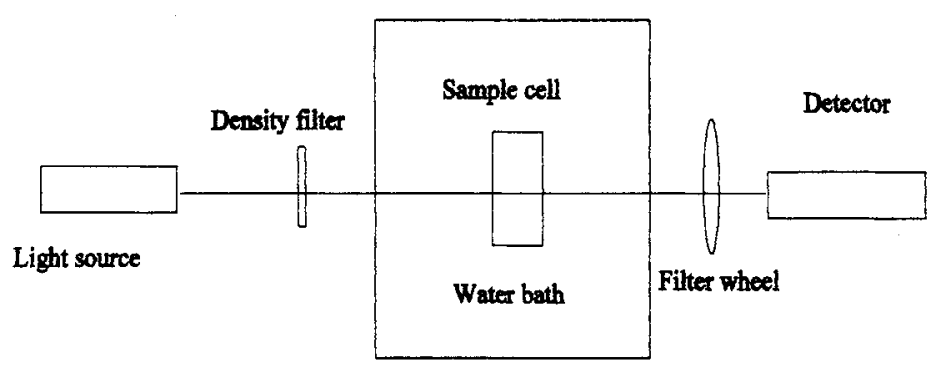

Fig. 3 Schematic diagram of apparatus for measurement of turbidity with $\alpha=2\left(2 n \pi \xi / \lambda_{0}\right)^{2}$. An optical system for measurement of turbidity is shown schematically in Fig. 3. A sample cell was clamped in a cell holder and placed in a water bath. The sample holder was attached to a precision stage with fine level adjustment and could be moved vertically. The stage was able to be moved side to side through a screw system. The light source may be a 
Xenon arc lamp or a He-Ne laser. The well- collimated light beam passed through a density filter before it transmitted the optical cell. A filter wheel was placed before a detector assembly, in which several narrowband-pass filters with various wavelengths were mounted. The detector assembly consisted of an aperture, an amplifier, and a photodiode or a photomultiplier tube (PMT). The size of the aperture, and the distance between the cell and the aperture were so arranged that a contribution to transmission intensity from scattered light including multiple scattering was negligible. The analogue voltage outputted by the detector assembly is proportional to the intensity of the transmission light. Two voltages with and without the optical cell in the light beam (defined as $V s$ and $V_{0}$ ) were measured alternatively by moving a cell side to side and repeated several times. An average value of $R\left(=V_{s} / V_{0}\right)$ was calculated. The turbidity then was obtained from:

$$
\tau=(-\ln R) / L-\tau_{b}
$$

where, $L$ is the cell path length, $\tau_{b}$ is the background turbidity determined by measurements of $R$ at the temperature far from the critical point. In principle, the exponents $n, g, \gamma$, and $v$ may be obtained, by using equations (1) and (5), from a series of measurements of turbidity at various temperatures and wavelengths. However, in practice, simultaneous determinations of these four exponents are often difficult because of the strong correlations between them (ref.9, ref.12). Therefore the values of $\gamma$ and $v$ were frequently fixed at their theoretical ones, and sometimes $\xi_{0}$ and $\chi_{0}$ were also related by a theoretical relation (ref.13, ref.14):

$$
R=\xi_{0}\left(B^{2} / 4 k_{b} T_{c} \chi_{0}\right)^{1 / 3}=0.65 \sim 0.67
$$

to avoid the large errors introduced from the correlations.

\section{COMPARISON BETWEEN THEORY AND EXPERIMENT}

\section{Critical yolume fraction}

A relation of volume fraction dependent on molar mass derived from Flory-Huggins theory (ref.3) may be expressed as:

$$
\phi_{c} /\left(1-\phi_{c}\right) \propto M^{-r}
$$

with $r=1 / 2$. For large $M$ of polymer solutions, $\left(1-\phi_{c}\right) \rightarrow 1$, equation (8) becomes:

$$
\phi_{c} \propto M^{-r}
$$

The experimental value of $r$ in equation (9) ranges from 0.37 to 0.40 and is generally believed to be universal to polymer solutions, but no theoretical explanations have been made for this universal value. The critical volume fractions for several mixtures of small chain molecules have been determined for dimethylacetamide (DMA) (ref.10), nitrobenzene (NB) (ref.15), diethyl maleate (DEM) (ref.16) in n-alkane with various chain length. The results and those of polystyrene (PS) in methylcyclohexane (MCH) (ref.17) and polymethylmethacrylate (PMMA) in 3-octanone (3-OCT) (ref.18) reported in literatures were analysed by using equation (8). TABLE 2 lists the values of $r$ associated with equation (8). It shows that equation (8) is universal and the value of $r$ is $0.41 \bullet 0.01$ for the chain-molecule solutions of both small molecules and polymers. Still no theories have been developed to explain this universality.

TABLE 2. Values of $r$ for Chain-Molecule Solutions

\begin{tabular}{ccccc}
\hline PS + MCH & PMMA + 3-OCT & DMA + n-alkane & NB + n-alkane & DEM + n-alkane \\
\hline $0.42 \pm 0.01$ & $0.40 \bullet 0.01$ & $0.41 \pm 0.01$ & $0.40 \pm 0.01$ & $0.42 \pm 0.01$ \\
\hline
\end{tabular}


Taking $\phi_{\mathrm{c}} /\left(1-\phi_{c}\right) \propto M^{-0.41}$, and combining equations (1) and (2) yield:

$$
\begin{array}{ll}
B\left(1-\phi_{c}\right)^{-1.865} \propto M^{-b}, & b=0.29 \\
\xi_{0}\left(1-\phi_{c}\right)^{0.85} \propto M^{n}, & n=0.18 \\
\chi_{0}\left(1-\phi_{c}\right)^{-1.20} \propto M^{g}, & g=-0.06
\end{array}
$$

\section{Exponent 6}

The coexistence curves of mixtures of (DMA + n-alkane) and (PMMA + 3-OCT) were determined by measurements of refractive indices in coexisting phases by the technique described above. The value of $b$ reduced from coexistence-curve data by using equation (10a) are $0.29 \pm 0.04$ for (DMA + n-alkane), and 0.26 \pm 0.03 for (PMMA $+3-\mathrm{OCT})$, respectively. The $\ln$-ln plots of $B\left(1-\phi_{c}\right)^{-1.865}$ against $M$ for these two systems are shown in Fig.4 and Fig.5. The experimental coexistence-curve data of (PS + MCH) reported by Dobashi et al. (ref. 15) are also reanalysed to obtain $n=0.30 \pm 0.02$. As a comparison, Fig. 6 shows a $\ln$ - $\ln$ plot of $B$ against $M$ for (PMMA + 3-OCT) (See equation (1a)), in which an experimental point with low molar mass is significantly off a straight regression line.

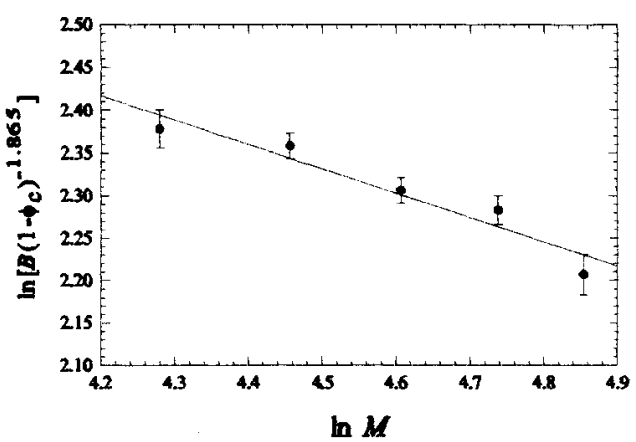

Fig. 4. In-ln plot of $\left[B\left(1-\phi_{\mathrm{c}}\right)^{-1.865}\right]$ against $M$ for DMA + n-alkane

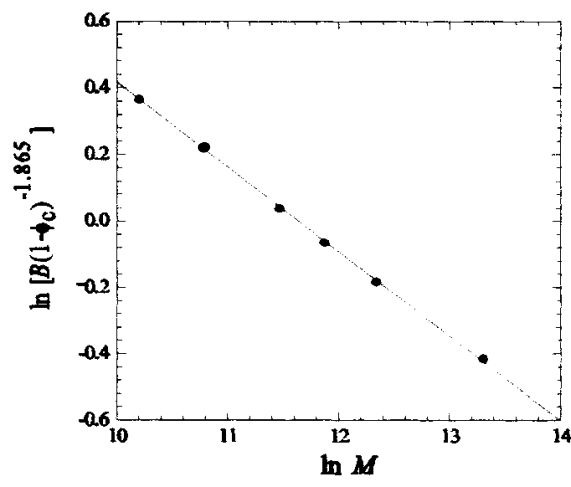

Fig. 5. In-ln plot of $\left[B\left(1-\phi_{c}\right)^{-1.865}\right]$ against $M$ for PMMA + 3-OCT

\section{Exponent n}

The correlation lengths of (PS + MCH) were determined by Shinozaki et al. (ref.8) for six samples of polystyrene with different molar mass. A $\ln -\ln$ plot of $\xi_{\theta}$ against $M$ (See equation (1b)) shows a discontinuous "step" ( See Fig. 7). Shinozaki et al. deleted two experimental points with small molar mass and obtained $n=0.28 \pm 0.03$. However, a value of 0.23 may be obtained, if all of the six points are equally

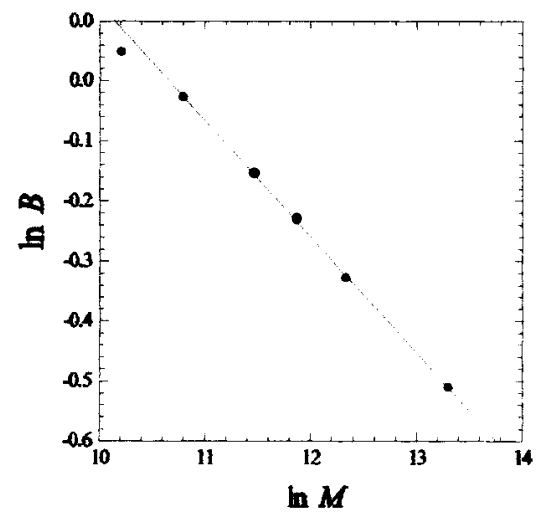

Fig. 6. In-ln plot of $B$ against $M$ for PMMA + 3-OCT

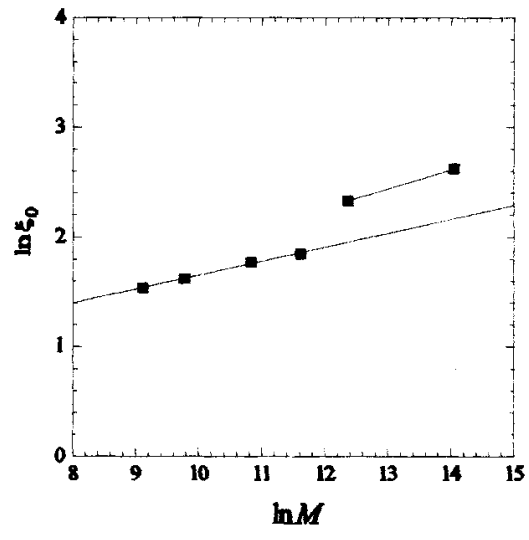

Fig. 7. In-In plot of $\xi_{o}$ against $M$ for PS + MCH 
weighted in data reduction. We reanalysed four experimental points with lower molar mass and two experimental points with higher molar mass separately by using equation $(1 b)$, and obtained $n=0.13$ and 0.17 , respectively (See straight regression lines in Fig. 7). Separate analyses of two parts of experimental points for the same system by using equation (10b).) gave the values of 0.18 and 0.19 for $n$, respectively, which are consistent with the theoretical value of 0.19 . Very recently, a series of turbidity measurements for (NB + n-alkane) was conducted in our laboratory to obtain the critical amplitudes associated with the correlation length and susceptibility (ref.19). A $\ln$ - In plot of $\xi_{d}\left(1-\phi_{c}\right)^{0.85}$ against $M$ gives a value of $0.21 \pm$ 0.04 for $n$, which is also consistent with the theoretical value.

\section{Exponent g}

Few precise susceptibility data are available for our analysis. A In-ln plot of $\chi_{0}\left(1-\phi_{c}\right)^{-1.20}$ against $M$ ( See equation (10c)) for (NB + n-alkane) gave a value of $-0.12 \pm 0.09$ for $g$, which is consistent with the theoretical value, but with less confidence because of the large errors in $g$.

In conclusion, equation (10) derived from the LGW model without the limitation of large $M$ is satisfied for the chain-molecule solutions of both small molecules and polymers. Further experimental studies on the critical behaviours of various chain-molecule solutions will be carried on to test this kind of universality.

This work was supported by the National Natural Science Foundation, the State Education Committee, and the Natural Science Foundation of Gansu Province, P. R. China.

\section{REFERENCES}

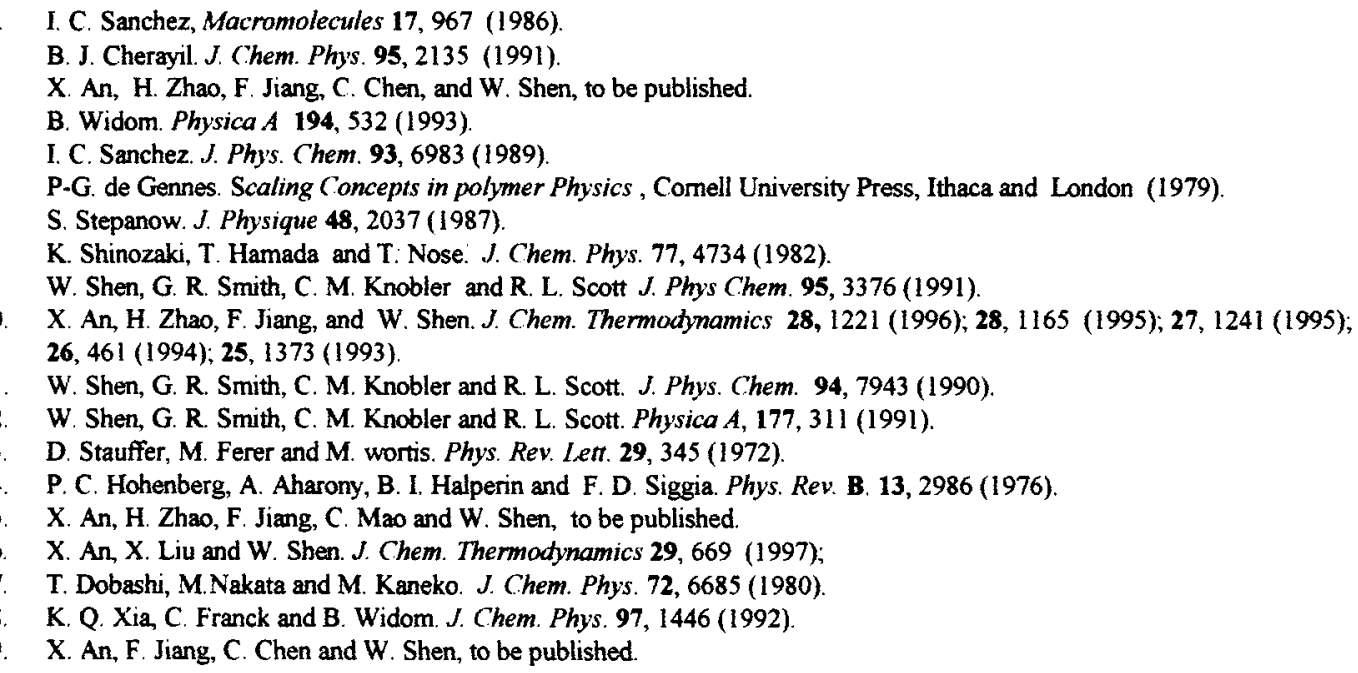

